\title{
Les caractéristiques architecturales et constructives de la muraille médiévale à la période Hammadite à Bejaia (Algérie)
}

The architectural and constructive characteristics of the medieval wall of Bejaia city during the Hammadite period

\section{Naima Abderrahim Mahindad}

Institut d'Architecture et Urbanisme - Laboratoire Environnement et Technologie pour l'Architecture et le Patrimoine - Université Saâd Dahleb, Blida 1, Algeria, mahindadnaima@gmail.com

\begin{abstract}
Bejaia, is a coastal city of Central-East Algerian which has seen succeeding on its lands several civilizations: Byzantine, Roman, Hammadite, Spanish and Ottoman.

It reached its peak from the beginning of the eleventh century, when the Hammadite ruler, An-Nasir made it the capital. The city maintained this important status until the sixteenth century, when it was considered the jewel of the Maghreb.

At that time, the city was fortified with a large surrounding wall, which spanned more than $5000 \mathrm{~m}$. This city wall was flanked with bastions and towers, and rose in tiers from the sea-side to Mount Gouraya. Its layout was perfectly designed and blended with the city's topography. It consisted of three walls: one to the east and another to the west, which were connected by a third wall, which ran along the seaside.

Today, two gates are preserved from the city of Bejaia's rich defensive heritage: Bab El Bahr, which opens onto the sea, and Bab El Fouka, which opens onto the plains, as well as some parts of the walls, dotted around different parts of the city. This heritage is threatened and its preservation, restoration and enhancement require a comprehensive knowledge of the architectural and constructive styles, which characterize it, and of the materials used in its construction.

This contribution aims to identify the architectural and constructive features of this defense system, developed by the Hammadites, as well as a characterization of the construction materials used, such mortar, through physical, chemical and petrographic analyses.
\end{abstract}

Keywords: Bejaia, Hammadite period, architectural characteristics, characterization of the materials, constructive typologies.

\section{Introduction}

La connaissance des bâtiments historiques doit reposer sur la connaissance de leurs matériaux et de leurs systèmes constructifs. Connaître les caractéristiques architecturales et constructives du système défensif des hammadites à la période médiévale et les caractéristiques de leurs maté- riaux constructifs est une source d'information importante pour comprendre l'évolution historique et archéologique de la construction de ce système défensif.

Dans le cas de l'Algérie, les études relatives aux matériaux et aux systèmes de construction sont 
récentes. Les fortifications de l'époque hammaditte en Algérie et particulièrement dans la ville de Bejaia entre 1067 et 1509 ont fait l'objet de très peu d'études.

$\mathrm{Au}$ cours des dernières décennies, les recherches sur les liants de maçonnerie ont essentiellement porté sur la caractérisation des matériaux utilisés dans les bâtiments historiques (PalazzoBertholon, 1998; Coutlas, 2003).

Le premier objectif de cette étude est d'identifier les différents systèmes constructifs adoptés dans la muraille Hammadites et notamment dans les deux portes qui subsistent à Bejaia et les différents matériaux utilisés à cette époque. Le deuxième objectif est de caractériser les anciens mortiers à base de chaux, utilisés dans cette fortification, et d'identifier leur composition chimique et minéralogique, ainsi que leurs techniques de production.

\section{Description architecturale de la muraille Hammadite}

A partir de l'année 1067, où la ville devint la capitale Hammadite, la ville a été protégée par une nouvelle enceinte. Elle était flanquée de bastions qui, du bord de la mer s'élèvent par gradins et vont se perdre dans les rochers abrupts du mont Gourraia (Feraud, 1869).

Elle est constituée de deux murailles qui partent du bord de la mer et s'étendent à l'est et à l'ouest, définissant un rectangle de 140 à 150 ha (Carette, 1848). Le mur a une étendue de 5400 $\mathrm{m}$, alors que les indications concernant les dimensions de sa largeur et sa hauteur varient d'un auteur à l'autre: La largeur varie de $90 \mathrm{~cm}$ à 2,50 m (Beylié, 1909; Isnard, 1954) et la hauteur varie de 4 à $8 \mathrm{~m}$ (Vivien, 1834).

C'est un mur crénelé, couronné par un chemin de ronde. L'enceinte est renforcée par le flanquement de tours pleines à base rectangulaire de $4 \mathrm{~m}$ sur 3 à la base et de $6 \mathrm{~m}$ de hauteur (De Beylié, 1909). Par contre sur la face orientale du mur, on voit des saillants barlongs de 2,50 m de saillies sur $4 \mathrm{~m}$ de large, distants l'un de l'autre d'environ $25 \mathrm{~m}$.

Les communications de la ville avec l'extérieur se faisaient à travers les différentes portes qui perçaient l'enceinte et qui étaient au nombre de six (06) : Bâb El-Bahr : L'unique porte qui a survécu aux guerres et aux temps. (Brunschving, 1940), se trouve dans le rempart sud longeant la mer. Bâb Amsiouen : sur le rempart est, à moitié route de Sidi Yahia (El Ghobrini, 1970). Bâb El-Marsa : Dans la région de Bridja inférieure (Brunschving, 1940). Bâb El-Bunud ou "la porte des Etendars", actuelle porte Fouka, se situe au nord-ouest de la ville (De Beylié, 1909). Bâb ElLûz: "porte des amandiers" était à l'ouest (El Ghobrini,1970). Bâb El-Mergoum: "porte de la compagne" que mentionne Ibn Khaldûn, située à hauteur de la Koubba de Sîdi Mohamed Amokran, à la tête du chemin Roman qui, mène au fort Clauzel (Ibn Khaldoun, 1927). Bâb Dar EsSanâa : "la porte de la Darse" (Feraud, 1869), elle disparut après la prise de Bejaia par les Espagnols, lors de la reconstruction de la Qasabah.

De toutes ces portes, la ville n'en a conservé que deux, vu l'importance du rôle qu'elles jouaient : Bâb El-Bahr (porte Sarazine) et Bâb El-Bunud (porte Fouka) et qui feront l'objet de notre étude (Fig. 1).

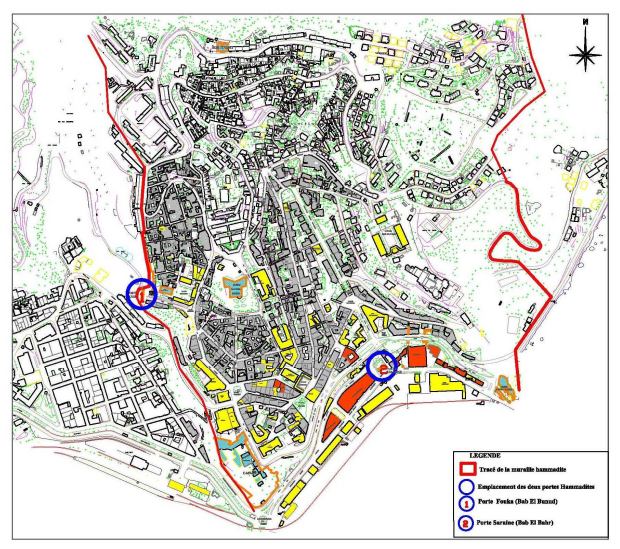

Fig. 1. Plan de situation de la muraille Hammadite (Abderrahim, 2019).

\subsection{La porte Fouka (Bâb el Bunud)}

C'était une porte monumentale (Fig. 2). Elle est garnie de grandes lames et elle est encadrée par deux bastions (De Beylié, 1909). A l'origine, c'était une porte flanquée de deux tourelles pentagonales formant une saillie, dont la fonction 
est de la protéger, l'entrée et son passage y sont encastrés.

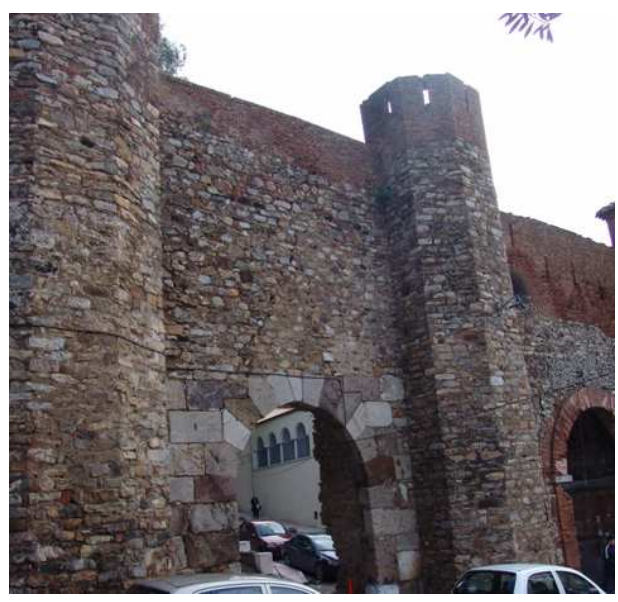

Fig. 2. Vue génerale la porte Fouka (Abderrahim, 2015).

Elles sont composées de couches alternatives de moellons et de pierres et de briques. Ces tours sont surmontées de meurtrières en briques plates, dépassant le mur de $2 \mathrm{~m}$. La porte est composée d'un arc plein cintre construit en pierres surmonté d'un mur épais appareillé de lits de moellons alignés, alternant des pierres plates et des briques en terre cuite. Cette maçonnerie est surmontée d'un chemin de ronde. A l'époque française cet ouvrage a été fortement transformé : il y a eu percement d'une nouvelle porte à proximité de l'ancienne, au niveau de la troisième tourelle qui est de forme carrée.

\subsection{La porte Sarazine (Bâb El Bahr)}

C'est une porte non flanquée, établie dans l'épaisseur de la courtine. Les deux murs flanquant qui forment deux avancées en grande partie ruinée, jouaient sans doute le rôle de contreforts destinés à assurer, la solidité de la porte qui baignait dans la mer. Ils sont composés de couches alternatives de briques et de moellons.

Vue de mer, la porte offre sur sa façade le tracé d'un arc brisé (Fig. 3) qui assure la cohésion de la voûte.

Cet arc est entièrement composé de briques plates, pleines, disposées en lits horizontaux jusqu'à une hauteur de 1,50 m environ, puis un arc rayonnant pour former la voûte, il repose sur des pieds droits en retrait sur la retombée d'arc. Un second arc brisé parallèle et semblable au premier, composé de briques plates convenablement alignées, forme un décrochement sur la façade de la porte et supporte une lourde maçonnerie que devait surmonter le chemin de ronde (Golvin, 1957).

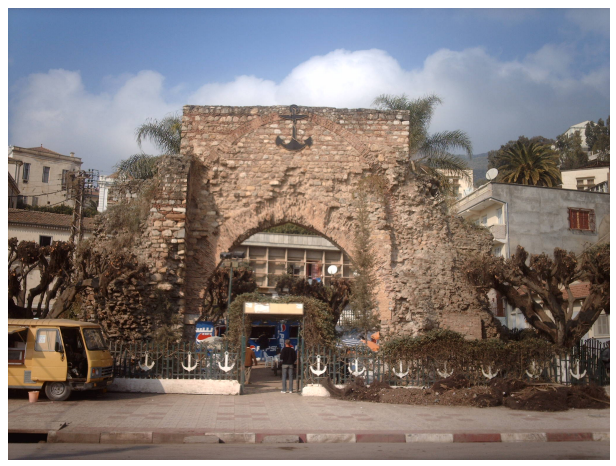

Fig. 3. Vue génerale de la porte Sarazine (Abderrahim, 2015).

\section{Les typologies constructives}

Les matériaux utilisés dans la construction des deux portes "Porte Fouka" et "Porte Sarazine" sont les moellons et la brique.

Le matériau le plus prédominant dans l'appareillage des murs est le moellon, alors que les arcs et les couvertures maçonnées sont réalisés en briques hourdées au mortier de terre ou de chaux.

On relève deux systemes constructifs qui ont été adoptés dans la construction de la muraille hammadite et dans les portes qui y sont aménagées.

\subsection{Des murs en terre banchée}

Ils constituent les murs porteurs. Les murs porteurs en terre banchée sont constitués d'un parement qui alterne des lits de pierres, de moellons et de briques pleines posées à plat. C'est des parements en appareillage mixte (opus mixtum) avec blocage en moellons concassés, graviers, tuf et chaux. L'ensemble des maçonneries est hourdé au mortier de chaux. 
Néanmoins, on relève des différences dans l'appareillage des parements entre la porte Sarazine et la porte Fouka.

- Un mur en terre banché avec parement mixte régulier (Fig. 4), au niveau de la porte Sarazine : les deux parements sont constitués de pierres équarries alternée avec des lits de briques. Bien que le système ne soit pas totalement régulier, on décèle un rythme par bandes qui alterne généralement, deux assises de briques avec deux assises de pierres.

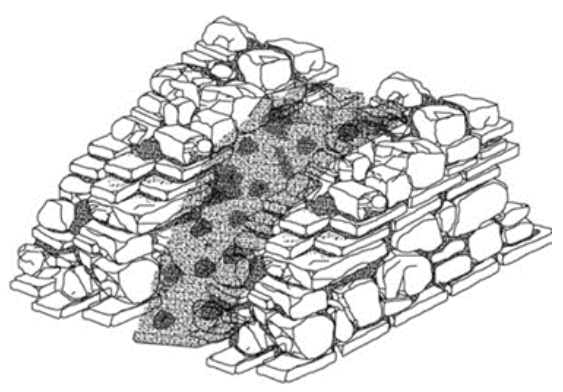

Fig. 4. Vue Axonométrique du mur en terre banché avec parement régulier (Abderrahim, 2016).

- Un mur en terre banché avec parement mixte irrégulier (Fig. 5), au niveau de la porte Fouka : il est constitué d'un parement qui alterne des lits de pierres équarries, de moellons et de briques de façon disparate. Les briques pleines sont posées à plat (opus mixtum).

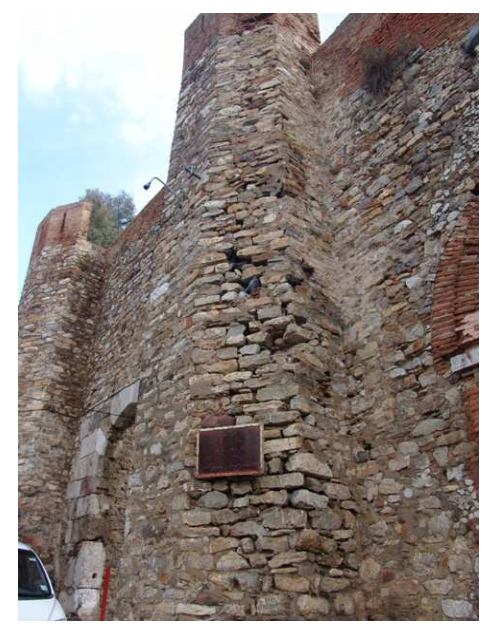

Fig. 5. Vue génerale du mur en terre banché avec parement irrégulier (Abderrahim, 2017).
Entre les deux parements, on trouve un blocage constitué de moellons concassés, du gravier, tuf et chaux. L'ensemble des maçonneries est hourdé au mortier de chaux.

\subsection{Des murs de commande mixtes}

Ce sont des murs épais réalisés en en appareillage mixte (moellons et briques) hourdés avec un mortier à base de terre et de chaux qui dépasse $1,10 \mathrm{~m}$. On trouve cet appareillage audessus du premier arc ogival et au niveau du mur transversal de la porte (Fig. 6 et Fig. 7).

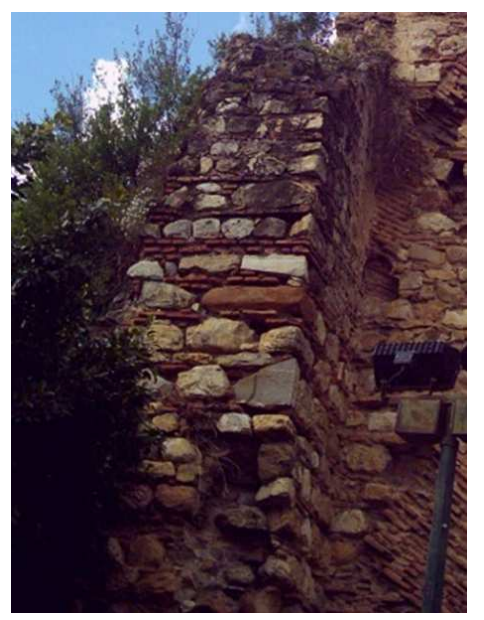

Fig. 6. Vue génerale du mur de commande mixte (Abderrahim, 2017).

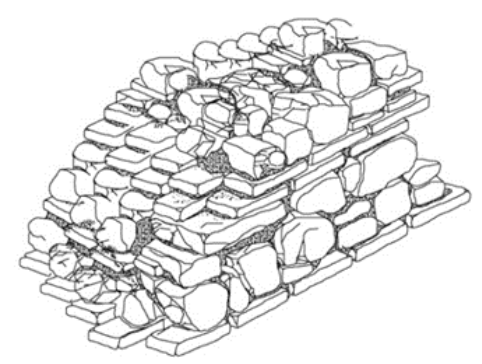

Fig. 7. Vue axonométrique du mur de commande mixte (Abderrahim, 2017).

La porte des étendards se distingue par un troisième système constructif, qui est constitué de murs épais réalisés en briques pleines et posés à plat et hourdés avec un mortier à base de terre et de chaux. On retrouve cet appareillage surtout au niveau du chemin de ronde et des meurtrières 
(Fig. 8). Cette typologie constructive est plus tardive et date de la période française.

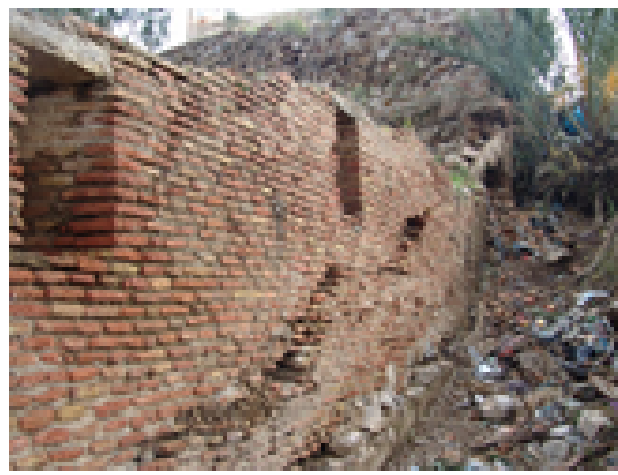

Fig. 8. Vue générale du mur de commande en briques (Abderrahim, 2017).

\section{Caractérisation des mortiers de la période Hammadite}

Des echantillons de mortiers de jointoiement ont été prélevés dans chacune des deux portes

Les prélèvements de mortier ont été d'abord comparés sur la base de critères macroscopiques Il s'agit de l'observation des échantillons à l'œil nu, sans préparation particulièr (PalazzoBertholon, 2000).

Ces premières observations ont été complétées par une étude pétrographique et des analyses physico-chimiques qui ont permis la caractérisation des mortiers hammadites et la détermination de leur caractéristiques.

\subsection{Description macroscopique des échantil- lons de mortiers}

Les échantillons de mortiers de jointoiement, prélevés dans des édifices de la ville de Bejaia, ont des nuances de couleurs relativement claires. Elles varient du beige gris au jaune orangé. A l'œil nu, on peut observer certains composants qui se distinguent par leurs couleurs et leurs granulométries. Les deux échantillons MJPF (Porte Fouka) et MJPS (Porte Sarazine) sont de de couleur beige gris, friables à la main.

Le mélange est homogène et présente une granulométrie très fine des composants. On relève aussi la présence de pores et de traces de chaux.

\section{2. Étude pétrographique des échantillons}

Deux échantillons de mortiers ont été choisis pour être préparés en lame mince, sur la base de la typologie macroscopique. L'étude des lames minces permettent de préciser les observations faites sur les échantillons bruts, en particulier la liaison de la charge avec la matrice et la nature pétrographique des grains de la charge. Cette étude pétrographique a été réalisée selon le protocole adopté en géologie pour l'étude des différents types de roches (Boulevain, 2015).

\section{a) Échantillon MJPF (Porte des étendars)}

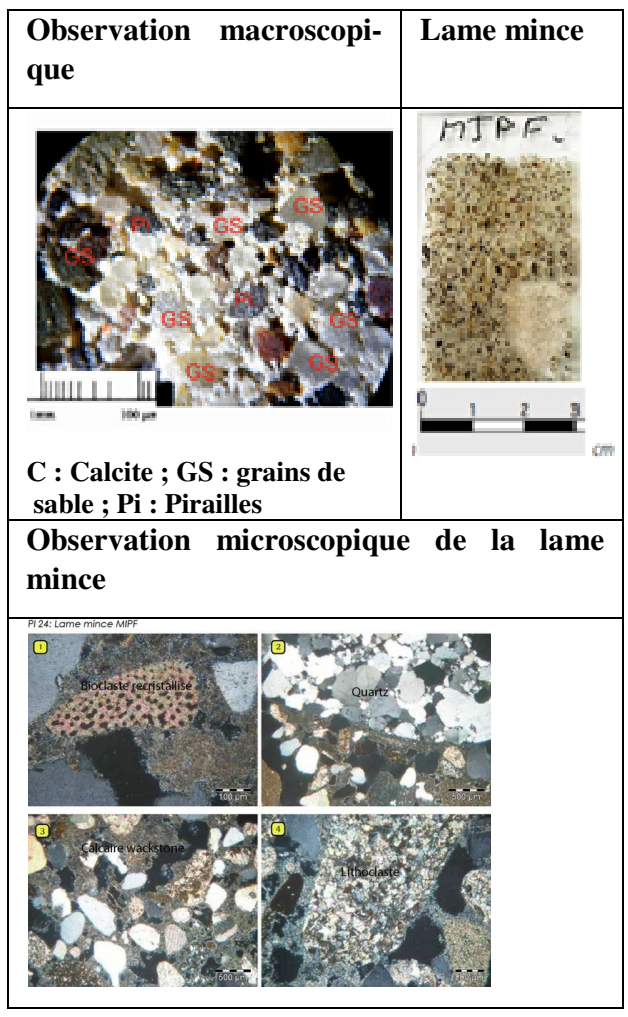

\section{Description et interprétation}

La matrice est une boue carbonatée, très peu abondante, très fine et homogène, de couleur beige et, parsemée d'éléments épars foncés de couleur noire et brun. C'est une texture Wackstone dont la phase granulaire est constituée de gros clastes gréseux constitués de quartz monocristallin et poly cristallin, des lithoclastes, des plagioclases, des nodules de dolomite oxy- 
dée, des bioclastes et de quelques pelletoides. La porosité de dissolution et de rétraction n'est pas très importante, elle est de moins de $10 \%$.

\section{b) Échantillon MJPS (Porte Sarazine)}

\begin{tabular}{|l|l|}
\hline $\begin{array}{l}\text { Observation macroscopi- } \\
\text { que }\end{array}$ & Lame mince \\
\hline & \\
& \\
\hline
\end{tabular}

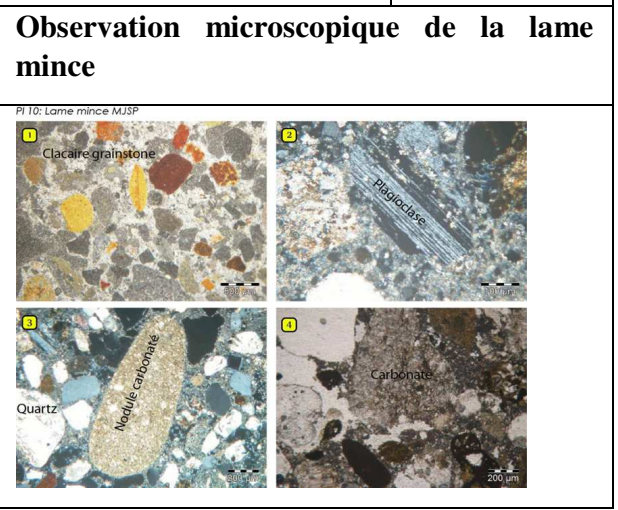

\section{Description et interprétation}

La matrice est une boue carbonatée, très peu abondante, très fine et homogène, de couleur beige et, parsemés d'éléments épars foncés de couleur noire et brun. C'est un calcaire à texture wackstone à pelletoide, avec des éléments jointifs et un liant très peu abondant. La phase granulaire est constituée d'agrégats, de calcite, de quartz monocristallin et polycristallin, d'argile et de l'oxyde de fer. L'argile se présente sous forme de grains issus probablement de l'altération des feldspaths (kaolinite et illite). $S$ 'ajoute à cela quelques foraminifères planctoniques. La porosité de dissolution et de rétraction n'est pas très importante, elle est de moins de $10 \%$.

\subsection{Caractéristiques physiques des échantil- lons}

Les échantillons prélevés ont fait l'objet de plusieurs essais physiques dont l'ensemble des resultats sont reportés dans le tableau 1.

\begin{tabular}{|l|l|l|}
\hline & $\begin{array}{l}\text { Porte } \\
\text { Fouka } \\
\text { MJPF }\end{array}$ & $\begin{array}{l}\text { Porte Sara- } \\
\text { zine } \\
\text { MJPS }\end{array}$ \\
\hline $\begin{array}{l}\text { Masse volumique } \\
\text { apparente g/cm }\end{array}$ & 1,38 & 1,27 \\
\hline $\begin{array}{l}\text { Masse volumique } \\
\text { spécifique g/cm }\end{array}$ & 1,95 & 1,75 \\
\hline $\begin{array}{l}\text { Porosité totale } \\
\text { Pt (\%) }\end{array}$ & 29,23 & 27,42 \\
\hline $\begin{array}{l}\text { Absorption d'eau } \\
\text { Ab (\%) }\end{array}$ & 21,18 & 20,18 \\
\hline Humidité H (\%) & 2,45 & 2,25 \\
\hline $\begin{array}{l}\text { Chaux libre CaO } \\
\text { (\%) }\end{array}$ & 2,24 & 2,24 \\
\hline $\begin{array}{l}\text { Potentiel } \\
\text { HydrogènepH(\%) }\end{array}$ & 8,60 & 9,20 \\
\hline $\begin{array}{l}\text { Matières Organi- } \\
\text { ques Cmoc (\%) }\end{array}$ & 3,22 & 1,24 \\
\hline
\end{tabular}

Tab. 1. Résultats des analyses physiques.

\subsection{Analyse minéralogique (DRX)}

L'analyse minéralogique des échantillons de mortiers de jointoiement prélevés au niveau des deux portes Hammadides a permis d'obtenir les résultats présentés dans le tableau 2.

\begin{tabular}{|l|l|l|}
\hline Minéraux & $\begin{array}{l}\text { Porte Fouka } \\
\text { MJPF }\end{array}$ & $\begin{array}{l}\text { Porte Sara- } \\
\text { zine MJPS }\end{array}$ \\
\hline Quartz $\left(\mathbf{S i O}_{2}\right)$ & 31 & 15 \\
\hline $\begin{array}{l}\text { Albite } \\
\left(\mathbf{N a}_{2} \mathbf{A l S i}_{\mathbf{6}} \mathbf{O}_{\mathbf{1 6}}\right)\end{array}$ & 03 & 04 \\
\hline $\begin{array}{l}\text { Gypse }\left(\mathbf{C a S O}_{4}\right. \\
\left.\mathbf{2 H}_{\mathbf{2}} \mathbf{O}\right)\end{array}$ & 01 & 01 \\
\hline Calcite $\left(\mathbf{C a C O}_{3}\right)$ & 51 & 66 \\
\hline $\begin{array}{l}\text { Kaolinite } \\
\text { Al }\end{array} \mathbf{S i}_{2} \mathbf{O}_{\mathbf{5}}\left(\mathbf{O H}_{\mathbf{4}} \mathbf{4}\right.$ & 08 & - \\
\hline Hématite $\left(\mathbf{F e O}_{3}\right)$ & 06 & 05 \\
\hline Périclase $\mathbf{M g O}$ & - & 08 \\
\hline Autres & 02 & 02 \\
\hline
\end{tabular}

Tab. 2. Résultats analyse minéralogique.

Le principal composant pour l'ensemble des échantillons est la calcite dans des proportions 
différentes entre les deux echantillons MJPS et MJPF: Le mélange des différents mortiers s'est fait dans des rapports calcite/quartz, qui dépassent 3,25 pour l'échantillon MJPS, soit de 3 à 4 parts et demi de chaux pour une part de sable alors que l' échantillons MJPF , présente un rapport calcite/quartz moins important entre 1,64 à 1,84 , soit presque deux parts de chaux pour une part de sable.

\subsection{Analyse chimique}

L'analyse chimique des deux echantillons prélevés a été réalisée selon le principe de la norme NF P 15-467, alors que l'indice d'hydraulicité a été calculée selon l'équation suivante (Boynton, 1980).

$$
H I=\frac{\mathrm{Al} 203 \%+\mathrm{Fe} 203 \%+\mathrm{SiO} 2 \%}{\mathrm{CaO} \%+\mathrm{MgOA} \%}
$$

L'ensemble des résultats sont reportés dans le tableau 3.

\begin{tabular}{|l|l|l|}
\hline $\begin{array}{l}\text { Echantillons } \\
\text { Teneurs }\end{array}$ & $\begin{array}{l}\text { Porte Fouka } \\
\text { MJPF }\end{array}$ & $\begin{array}{l}\text { Porte Sarazine } \\
\text { MJPS }\end{array}$ \\
\hline $\mathbf{S i O}_{2}$ & 37,59 & 19,81 \\
\hline $\mathbf{A l}_{\mathbf{2}} \mathbf{O}_{3}$ & 3,56 & 3,99 \\
\hline $\mathbf{F e}_{\mathbf{2}} \mathbf{O}_{\mathbf{3}}$ & 3,91 & 3,71 \\
\hline $\mathbf{C a O}$ & 28,36 & 36,93 \\
\hline $\mathrm{MgO}$ & 1,36 & 1,59 \\
\hline $\mathbf{S O}_{\mathbf{3}}$ & 0,60 & 0,62 \\
\hline $\mathbf{K}_{\mathbf{2}} \mathbf{O}$ & 0,37 & 0,68 \\
\hline $\mathbf{N a}_{\mathbf{2}} \mathbf{O}$ & 0,35 & 0,53 \\
\hline $\mathbf{P}_{\mathbf{2}} \mathbf{O}_{\mathbf{5}}$ & 0,09 & 0,14 \\
\hline $\mathbf{T i O}_{\mathbf{2}}$ & 0,16 & 0,16 \\
\hline $\mathbf{P F}$ & 23,57 & 31,85 \\
\hline $\mathbf{H I}$ & 1,51 & 0,72 \\
\hline
\end{tabular}

Tab. 3. Résultats des analyses chimiques.

Ces résultats démontrent que le plus important composant, dans l'échantillon MJPS, est le $\mathrm{CaO}$ avec des teneurs de $36,93 \%$.et une teneur moins importante en en $\mathrm{SiO}_{2}$. Par contre l'échantillon et MJPF, a une teneur en $\mathrm{SiO}_{2}$, plus importantes que celle en $\mathrm{CaO}$. L'échantillon MJPS a une teneur de $37,59 \%$ en $\mathrm{SiO}_{2}$ pour une teneur de $28,36 \%$ en $\mathrm{CaO}$.
Par ailleurs, on remarque que la somme des oxydes $\mathrm{SiO}_{2}, \mathrm{Al}_{2} \mathrm{O}_{3}$ et $\mathrm{Fe}_{2} \mathrm{O}_{3}$ est plus élevée pour l'échantillon MJPF, par rapport à MJPS.

\section{Interprétation générale}

L'étude pétrographique, nous a permis d'identifier la texture des mortiers étudiés. Les mortiers étudiés MJPS et MJPF ont une texture "graveleuse" : ils contiennent une grande proportion de grains jointifs et une boue peu abondante. En plus du quartz et de la calcite, d'agrégats et de clastes gréseux, ces mortiers contiennent aussi des foraminifères planctoniques et des matières organiques.

L'analyse chimique fait ressortir dans la composition de l'échantillon MJPF (porte Fouka), des teneurs importantes de Silice dont l'origine peut être le sable quartzeux. Par ailleurs la teneur en $\mathrm{CaO}$, est moins importante dans cet échantillon, alors que pour l'échantillon MJPS (porte Sarazine), les teneurs en silice et en oxyde de calcium sont inversées. Les autres composants $\mathrm{Al}_{2} \mathrm{O}_{3}, \mathrm{Fe}$, Na et $\mathrm{K}$ sont présents en quantités beaucoup plus modérées.

L'analyse DRX des mortiers fait ressortir une particularité dans la composition des mortiers de jointoiement, que nous avons prélevés. C'est la présence de sulfate de calcium "le gypse" à des teneurs différentes. La présence du gypse pourrait etre expliqué par une contamination du mortier lors de sa préparation.

On relève la présence de Kaolinite dans l'échantillon de Bâb El Fouka, ce qui implique que ce mortier a été enrichi par des rajouts de fragments de briques broyées.

Le mélange des différents mortiers s'est fait dans des rapports Calcite/quartz qui dépassent 3,25 pour MJPS, soit de 3 à 4 parts et demi de chaux pour une part de sable. L'échantillon MJPF de la porte Fouka, présente un rapport calcite /quartz moins important entre 1,64 à 1,84, soit presque deux parts de chaux pour une part de sable.

A partir de ces compositions, nous avons pu calculer l'indice d'hydraulicité de chaque échantillon. Les résultats obtenus font apparaitre que ces 
indices ne sont pas très élevés variant de 0,72 à 1,51 .

\section{Conclusion}

Les compositions minéralogiques et chimiques des différents échantillons de mortiers étudiés, permettent l'identification des matières premières qui ont été utilisées pour leurs fabrications.

Le mortier de la porte Fouka est moins riche en calcite que l'echantillons de la porte Sarazine par contre il presente un meilleur índice d'hydraulicité $(1,51)$. Ce qui peut s'expliquer par la présence de Kaolinite qui est indicatrice de l'utilisation de la brique pillées dans la fabrication du mortier MJPF.

Les mortiers utilisés à Bejaia dans la muraille hammadite sont fabriqués avec les memes matériaux. Ce sont des mortiers riches en calcite mais qui différents dans les proportions utilisées et les matériaux rajoutées pour rendre le mortier plus performant comme la brique pillée.

\section{Bibliography}

Beylié, G.L. de. (1909). La Kalaa des Béni-Hammad, une capitale de l'Afrique du Nord, Paris, p. 105.

Boulevain, F. (2015). Identification microscopique des principaux constituants des roches sédimentaires (Notes et Travaux pratiques).

Brunschving, R. (1940-1947). La Berbèrie orientale sous les Hafsides de l'origine à la fin du XI siècle, tI et II, Maisonneuve, Paris, p. 378.

Carette, E. (1848). Etude sur la Kabylie, exploration scientifique de l'Algérie de 1840 à 1842, 2 vol., Paris, p. 13.

Coutelas, A. (2003). Petro archéologie du mortier de chaux gallo-romain, Essai de reconstitution et d'interprétation des chaines opératoires : Du matériau au métier antique, Thèse de Doctorat, Université Paris I, Panthéon Sorbonne, Paris.

El- Ghobrini, A. (1970). Unwân Ed-Dirâya, R. Bounar, p. 151.

Feraud, CH. (1896). Histoire des villes de la province de Constantine, L. Arnolet, Bougie-Constantine, p. 148.

Golvin, L. (1957). Le Maghreb central à l'époque des Zirides, Paris, p. 193.

Ibn Khaldoun. (1927). Histoires des Berbères t III, De Slane, trad., librairie orientaliste Geuthner, Paris 1927, p. 39.

Isnard, H. (1954). L'Algérie, Arthand, Paris, p. 79.

Palazzo-Bertholon, B. (2000). "Le dépôt archéologique, conservation et gestion pour un projet scientifique et culturel. Assises nationales de la conservation archéologique", Actes des Assises nationales de Bourges, 26-28 novembre 1998, Bourges, pp. 223-230.

Palazzo-Bertholon, B. (2013). Rapport d'etude des mortiers de l'eglise de san parteo, mariana (haute-corse), Projet strategique ACCESSIT, Programme de coopération transfrontalière Italie-France Maritime 2007-2013 Toscane Ligurie - Sardaigne - Corse, pp. 10-15. 